\title{
Morphological Characteristics of Nerve Cells in the Human General Sensory System
}

\author{
By
Tetsu YANAI, Noboru GOTO, Jun GOTO, Naoko NONAKA and Masakazu SHIBATA

Department of Anatomy, Showa University School of Medecine

- Received for Publication, January 24, 2005 -

Key Words: Neuron, K-B stain, Morphology, Sensory pathway

\begin{abstract}
Summary: We conducted a study to compare morphological characteristics of various nerve cells in the sensory system. This sort of evaluation is indispensable for a better understanding of the nervous system in relation to clinical physiology and neurology.
\end{abstract}

Several monographs ${ }^{1,2)}$ have been published concerning the morphology of the various brainstem nuclei in humans. Quantitative evaluations, however, are fairly rare, and, apart from our reports $^{3-8)}$, they have not used a staining method that minimizes the shrinkage ratio. We have conducted this study to bring to light the morphological characteristics of various neurons in the human general sensory system.

\section{Methods}

Using an image-analyzer equipped with a microscope and a special tissue preparation method (modified Klüver-Barrera method) $^{9)}$ to minimize the shrinkage ratio of sections, we observed nerve cells in the general sensory system: Spinal ganglia, Brodmann's Area 3 neurons (layers 4-6) in the postcentral cortex, neurons in the ventralis posteromedialis (VPM) and ventralis posterolateralis (VPL) nuclei in the thalamus, neurons of the Goll's and Burdach's nuclei in the posterior funicular nucleus, neurons in the sensorius principalis nucleus in the pons, neurons in the subnuclei zonalis, gelatinosus and magnocellularis of the pars caudalis in the trigeminal spinal tract nucleus, neurons in the cervical, thoracic, lumbar and sacral proprius posterior horn nucleus of the spinal cord.

\section{Results}

The shapes and sizes of the neurons mentioned above vary from nucleus to nucleus (see the figure). The spinal ganglia contain two types of neurons (Fig., SG): very large and medium size. Both are round, bipolar and contain fine Nissl bodies. Neurons are spindle-shaped and their sizes are very small in layer 4 of Brodmann's area 3 in the cerebral cortex of the postcentral gyrus (Fig., A3-4, A35, A3-6), in the subnucleus gelatinosus of the pars caudalis in the trigeminal spinal tract nucleus (Fig. STG), and in the cervical, thoracic, lumbar and sacral proprius posterior horn nuclei of the spinal cord (Fig., CPH, TPH, LPH, SPH), while they vary in shape (round, pyramidal, triangular, oval and spindle) with slight size differences in the other cases (Fig., VPM, VPL, G, B, SP, STZ, STM).

\section{Discussion}

Basically, a proper knowledge of the histologic organization of the nervous tissue is required to understand how the nervous system works. In the peripheral nervous system, neuronal cell-bodies are found in the spinal and trigeminal ganglia, while in

Correspondence: Noboru Goto, M.D., Department of Anatomy, Showa University School of Medecine, 5-8, Hayanodai 1, Shinagawa-ku, Tokyo 142-8555, Japan 


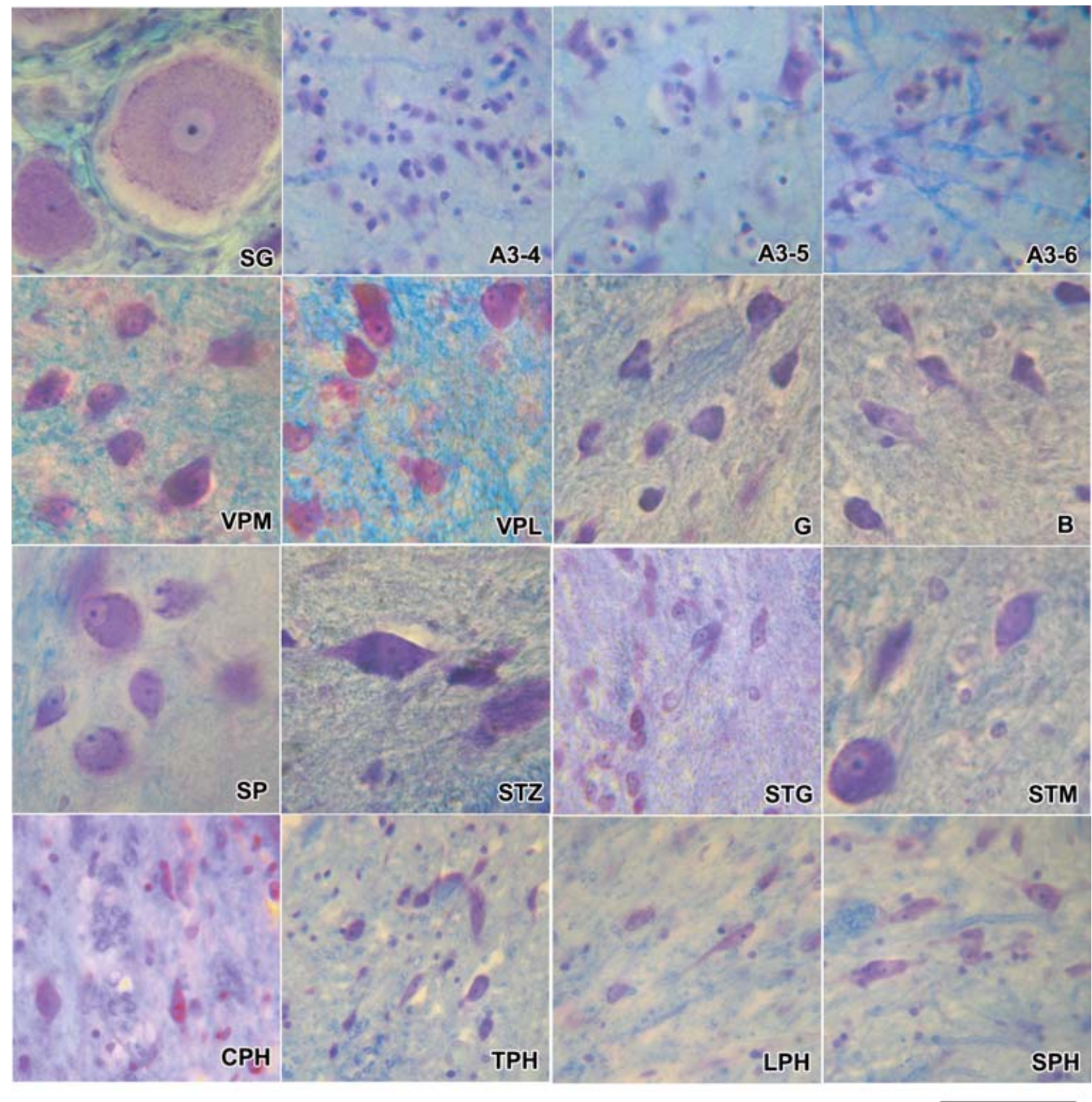

Fig. Representative neurons of the human general sensory system. Modified K-B stain, scale bar $=50 \mu \mathrm{m}$.

Abbreviations: SG: spinal ganglion; A3-4: area 3, layer 4; A3-5: area 3, layer 5; A3-6: area 3, layer 6; VPM: ventralis posteromedialis nucleus of the thalamus; VPL: ventralis posterolasteralis nucleus of the thalamus; G: gracilis nucleus of Goll; B: medial cuneate nucleus of Burdach; SP: principal sensory nucleus; STZ: zonal trigeminal spinal tract subnucleus; STG: gelatinous trigeminal spinal tract subnucleus; STM: magnocellular trigeminal spinal tract subnucleus; CPH: cervical posterior horn proprius nucleus; TPH: thoracic posterior horn proprius nucleus; LPH: lumbar posterior horn proprius nucleus; SPH: sacral posterior horn proprius nucleus.

the central nervous system, they are found in the nucleus proprius of the posterior horn at every level of the spinal cord; in the posterior funicular nuclei in the medulla oblongata (the gracil nucleus of Goll and the medial cuneate nucleus of Bur- dach); in the ventralis posteromedialis (VPM) and the ventralis posterolateralis (VPL) nuclei in the thalamus; and in the cerebral sensory corteces of Brodmann's areas 3,1 and 2.

It is interesting to note that there are different 
sizes and shapes of neurons within a single ascending pathway. For example, the posterior funiculusmedial lemniscus pathway contains spinal ganglion cells (SG), cervical to sacral proprius posterior horn cells $(\mathrm{CPH}, \mathrm{TPH}, \mathrm{LPH}, \mathrm{SPH})$, neurons in the posterior funicular nucleus $(G, B)$, neurons in the thalamus (VPL), and cerebral cortical neurons at Brodmann's area 3 (A3-4, A3-5, A3-6). This pathway contains neurons of three different sizes and shapes. However, the reason for this is not known.

Up to now, it had been difficult to compare the shape and size of neurons in the different nuclei with a sufficient degree of precision because of the wide ranges of the shrinkage ratios of tissue sections. One of the authors has devised anideal staining method for such purpose ${ }^{9)}$, including precise measurements: the modified Klüver-Barrera method which provides extremely precise data, with a shrinkage ratio of exactly $10 \pm 0 \%$ in length, and is most suitable for the comparison of different shapes and sizes.

An overall reevaluation of neuronal shapes and sizes is necessary to obtain a proper knowledge in order to better understand the structure of nervous pathways.

\section{References}

1) Olszewski J and Baxter D. Cytoarchitechture of the human brain stem. S. Karger, Basel-München-Paris-London-New York-Sydney, 1954 \& 1982.

2) Goto N. Atlas of the human brainstem and cerebellum. Medical Tribune, Tokyo, 1989 (in Japanese).

3) Setoyama S, Zhou M, Goto $\mathrm{N}$ and Shimada K. Morphometric comparison of the motor trigeminal neurons. Okajimas Folia Anat Jpn 1997; 73:301-312.

4) Yuan H, Ke M, Goto N, Goto J and Suzuki K. Morphometric evaluation of the human cervical motoneurons. Okajimas Folia Anat Jpn 2000; 76:277-284.

5) Yuan H, Goto N, Akita H, Shiraishi $\mathrm{N}$ and He H. Morphometric analysis of the human cervical motoneurons in the aging process. Okajimas Folia Anat Jpn 2000; 77:1-4.

6) Yuan H, Goto N, Akita H, Goto J and Jin S. Sexual dimorphism of the motoneurons in the human spinal cord. Okajimas Folia Anat Jpn 2000; 77:143-148.

7) Takaoki E, Nonaka N, Goto N, Suzuki M, Goto J and Shibata M. Morphometric comparison of human nerve cells: Special sensory system. Okajimas Folia Anat Jpn 2005; 81: (in press).

8) Umino M, Goto J, Goto N, Nonaka N and Shibata M. Morphometric comparison of human nerve cells: Pyramidal motor system. Okajimas Folia Anat Jpn 2005; 81: (in press).

9) Goto N. Discriminative staining methods for the nervous system: Luxol fast blue-periodic acid Schiff-hematoxylin triple stain and subsidiary staining methods. Stain Technol 1987; 62:305-315. 